\title{
铜催化的有氧氧化合成靛红类衍生物的方法
}

\author{
Ahmad, Muhammad Siddique 主亚敏郭云龙张赛赛沈增明* \\ (上海交通大学化学化工学院 上海 200240)
}

\begin{abstract}
摘要 研究了铜催化的脱羊环化生成靛红的方法, 使用氧气作为最终的氧化剂. 这个互补的 $\mathrm{C}\left(\mathrm{sp}^{3}\right)-\mathrm{H}$ 官能团化方法 为合成静红提供了一种新方案. $\mathrm{Cu} / \mathrm{O}_{2} / \mathrm{Co}$ 体系显示出良好的反应性和兼容性, 富电子和缺电子的官能团都可以很好地 兼容. 并基于机理研究和以前的文献报道, 提出了一个可能的假设机理.
\end{abstract}

关键词 铜催化; 碳氢键活化; 青定红; 脱羰; 氧气

\section{Copper-Catalyzed Aerobic Oxidation Strategy: A Concise Route to Isatin}

\author{
Ahmad, Muhammad Siddique Zhu, Yamin Guo, Yunlong \\ Zhang, Saisai Shen, Zengming* \\ (School of Chemistry and Chemical Engineering, Shanghai Jiao Tong University, Shanghai 200240)
}

\begin{abstract}
A copper-catalyzed decarbonylation cyclization to form isatins using oxygen as terminal oxidant is developed. This complementary way offers a new protocol for the synthesis of isatins through $\mathrm{C}\left(\mathrm{sp}^{3}\right)-\mathrm{H}$ bond functionalization in $\mathrm{Cu} / \mathrm{O}_{2} / \mathrm{Co}$ system. This system shows good reactivity and compatibility. Both electron-rich and electron-deficient functional groups can be tolerated. A postulated mechanism is proposed based on mechanistic studies and previous reports.
\end{abstract}

Keywords $\mathrm{Cu}$ catalyst; $\mathrm{C}-\mathrm{H}$ bond activation; isatin; decarbonylation; oxygen

\section{Introduction}

Isatins and their derivatives are a group of important structural units which have been found in many bioactive molecules. ${ }^{[1]}$ Isatins are also powerful building blocks for the construction of heterocyclic systems and spirocyclic compounds. ${ }^{[2]}$ Therefore, many methods have been developed for the synthesis of isatins. Traditionally, the synthesis of isatins was achieved through the methods developed by Sandmeyer, ${ }^{[3]}$ Stolle, ${ }^{[4]}$ and Martinet. ${ }^{[5]}$ However, these methods suffer from harsh conditions, poor yields and limited substrate choices. In view of this, new synthetic methods to overcome these drawbacks are still highly required. Inspiringly, some efficient methods have been found, such as aryne-based methods, ${ }^{[6]}$ transition-metal catalyzed oxidation, ${ }^{[7]}$ oxidative cleavage of carbon-carbon triple bond, ${ }^{[8]}$ sulfur ylide-mediated carbonyl homologation, ${ }^{[9]}$ iodine-mediated oxidation, ${ }^{[10]}$ and $\mathrm{C}-\mathrm{H}$ amination (Scheme 1). ${ }^{[11]}$ Although these methods showed some im- provements, there are still many problems like the requirement of heavy metal catalysts and excess amount of bases or strong oxidants and relatively low yields of products. Thus, the development of a concise and environmentally benign process to access isatins remains highly desirable. In $2010, \mathrm{Li}$ and co-workers ${ }^{[7 \mathrm{c}]}$ reported a significant and elegant example concerning copper-catalyzed preparation of isatins through intramolecular aromatic $\mathrm{C}-\mathrm{H}$ and aldehyde $\mathrm{C}-\mathrm{H}$ dual activation to construct $\mathrm{C}-\mathrm{C}$ bonds (Scheme 1). After that, Cheng and co-workers ${ }^{[7 \mathrm{~g}]}$ documented that 2-aminoacetophenones can undergo intramolecular cyclization to form isatins in the presence of a catalytic amount of copper salt under a balloon of $\mathrm{O}_{2}$ (Scheme 1). Inspired by these works, we envisioned a complementary way of $\mathrm{C}\left(\mathrm{sp}^{3}\right)-\mathrm{H}$ bond functionalization catalyzed by copper to form $\beta$-ketoamide core structure. Herein, a copper-catalyzed decarbonylation cyclization to form isatins using oxygen as terminal oxidant is reported.

* Corresponding author. E-mail: shenzengming@sjtu.edu.cn

Received March 14, 2019; revised May 28, 2019; published online June 12, 2019.

Project supported by the National Natural Sciences Foundation of China (No. 21672144).

国家自然科学基金(No. 21672144)资助项目. 
Previous work Sulfur ylide-mediated carbonyl homologation<smiles>[R]Nc1ccccc1C(=O)O</smiles><smiles>[R]Nc1ccccc1CC</smiles>
Oxidative cleavage of a carbon-carbon triple bond<smiles>[R]N(C(=O)C#C)c1ccccc1</smiles><smiles>C[N+](=O)[O-]</smiles>

(1) HBTU, DIPEA, $\mathrm{CH}_{2} \mathrm{Cl}_{2}$

(2) Oxone, r.t.

Transition-metal catalyzed oxidations<smiles>[2H]N(C(=O)C=O)c1ccccc1</smiles><smiles>[R]N(C(=O)CO)c1ccccc1</smiles>

[Pd or Fe or Ru]<smiles>C#CC</smiles>

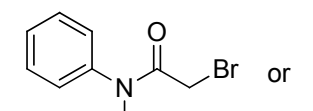<smiles>C=CC(=O)N(C)c1ccccc1</smiles>
$\mathrm{R}$ $\mathrm{R} \quad \mathrm{N}_{2}$<smiles>[R]Nc1ccccc1C(C)=O</smiles>
Cu/Oxidant

This work<smiles>[R]N(C=O)c1ccccc1C(C)=O</smiles>

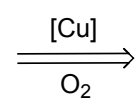<smiles>[R]N1C(=O)C(=O)c2ccccc21</smiles>

Scheme 1 Synthetic approaches for isatins

\section{Result and discussion}

To optimize reaction conditions, $N$-(2-acetylphenyl)- $N$ methylformamide (1a) derived from readily available 1-(2-aminophenyl)ethanone was chosen as a model substrate. Pleasingly, by using catalytic amounts of $\mathrm{CuCl}_{2}$ in the presence of THF and oxygen at $100{ }^{\circ} \mathrm{C}$, the conversion of 1a into the desired $N$-methyl isatine 2a was smoothly performed (34\% yield of 2a, Table 1, Entry 1). Next, a series of other copper catalysts was screened, such as $\mathrm{CuCl}, \mathrm{CuBr}, \mathrm{CuI}, \mathrm{CuSCN}, \mathrm{CuCN}, \mathrm{Cu}_{2} \mathrm{O}$ and $\mathrm{Cu}(\mathrm{OAc})_{2}$. The results exhibited that activity of these copper salts is inferiors to $\mathrm{CuCl}_{2}$ or even ineffective (Table 1, Entries 2 8). Using $\mathrm{CuCl}_{2}$ as the catalyst, a variety of Lewis acids were examined. A significant Lewis acid effect was observed. $\mathrm{CoCl}_{2}$ was the most effective Lewis acid for pro- moting formation of $\alpha$-ketoamide structure, affording $\mathbf{2 a}$ in $62 \%$ isolated yield (Table 1, Entry 16 ). $\mathrm{ZnCl}_{2}$ and $\mathrm{CoSO}_{4}$ slightly increased yield to $40 \%$ and $45 \%$, respectively (Table 1, Entries 9 and 15). Other Lewis acids, such as $\mathrm{AlCl}_{3}$, $\mathrm{BF}_{3} \bullet \mathrm{Et}_{2} \mathrm{O}, \quad \mathrm{FeCl}_{3}, \quad \mathrm{PdCl}_{2}, \mathrm{Cu}\left(\mathrm{ClO}_{4}\right)_{2} \bullet 6 \mathrm{H}_{2} \mathrm{O}, \mathrm{Yb}(\mathrm{OTf})_{3}$, $\mathrm{Ni}(\mathrm{OAc})_{2} \bullet 4 \mathrm{H}_{2} \mathrm{O}, \mathrm{Co}\left(\mathrm{NO}_{3}\right)_{2} \bullet 6 \mathrm{H}_{2} \mathrm{O}$ and $\mathrm{Cu}(\mathrm{OAc})_{2} \bullet 4 \mathrm{H}_{2} \mathrm{O}$ suppressed reaction and even shut down the process (Table 1, Entries $10 \sim 14$ and $17 \sim 20$ ). Interestingly, when 1 equiv. of protonic acid, $\mathrm{PhCOOH}$, combined with $\mathrm{CoCl}_{2}(1$ equiv.) was added into the reaction, the yield of $\mathbf{2 a}$ was increased to $69 \%$ (Table 1, Entry 24). To our surprise, when the loading of $\mathrm{CoCl}_{2}$ and $\mathrm{PhCOOH}$ was decreased to $20 \mathrm{~mol} \%$ catalytic amount, the efficiency of reaction was further improved and 78\% yield of 2a was obtained (Table 1, Entry 25). Notably, control experiment showed that $\mathrm{CoCl}_{2}$ could not promote this transformation in the absence 
of $\mathrm{CuCl}_{2}$ (Entry 26). Consequently, the optimal conditions involved the use of $\mathrm{CuCl}_{2}(20 \mathrm{~mol} \%), \mathrm{CoCl}_{2}(20 \mathrm{~mol} \%)$, $\mathrm{PhCOOH}(20 \mathrm{~mol} \%)$ in $\mathrm{O}_{2}$ and THF at $100{ }^{\circ} \mathrm{C}$.

Table 1 Optimization of reaction conditions for the synthesis of isatins $^{a, b}$<smiles>CC(=O)c1ccccc1N(C)C=O</smiles>

1a

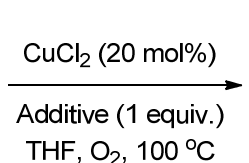

THF, $\mathrm{O}_{2}, 100^{\circ} \mathrm{C}$

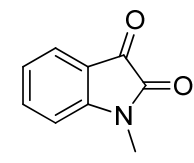

$2 a$

\begin{tabular}{|c|c|c|c|c|}
\hline Entry & Cat. & Additive & Time/h & Yield $^{b} / \%$ \\
\hline 1 & $\mathrm{CuCl}_{2}$ & None & 49 & 34 \\
\hline 2 & $\mathrm{CuCl}$ & None & 110 & 23 \\
\hline 3 & $\mathrm{CuBr}$ & None & 110 & 17 \\
\hline 4 & $\mathrm{CuI}$ & None & 82 & N.R. \\
\hline 5 & $\mathrm{CuSCN}$ & None & 82 & N.R. \\
\hline 6 & $\mathrm{CuCN}$ & None & 82 & N.R. \\
\hline 7 & $\mathrm{Cu}_{2} \mathrm{O}$ & None & 110 & Trace \\
\hline 8 & $\mathrm{Cu}(\mathrm{OAc})_{2}$ & None & 66 & N.R. \\
\hline 9 & $\mathrm{CuCl}_{2}$ & $\mathrm{ZnCl}_{2}$ (1 equiv.) & 49 & 40 \\
\hline 10 & $\mathrm{CuCl}_{2}$ & $\mathrm{AlCl}_{3}$ (1 equiv.) & 49 & N.R. \\
\hline 11 & $\mathrm{CuCl}_{2}$ & $\mathrm{BF}_{3} \cdot \mathrm{Et}_{2} \mathrm{O}$ ( 1 equiv. $)$ & 67 & N.R. \\
\hline 12 & $\mathrm{CuCl}_{2}$ & $\mathrm{FeCl}_{3}$ (1 equiv.) & 67 & N.R. \\
\hline 13 & $\mathrm{CuCl}_{2}$ & $\mathrm{PdCl}_{2}$ (1 equiv.) & 67 & N.R. \\
\hline 14 & $\mathrm{CuCl}_{2}$ & $\mathrm{Cu}\left(\mathrm{ClO}_{4}\right) \cdot 6 \mathrm{H}_{2} \mathrm{O}$ (1 equiv. $)$ & 67 & N.R. \\
\hline 15 & $\mathrm{CuI}$ & $\mathrm{CoSO}_{4}$ ( 1 equiv. $)$ & 59 & 45 \\
\hline 16 & $\mathrm{CuSCN}$ & $\mathrm{CoCl}_{2}$ (1 equiv.) & 59 & 62 \\
\hline 17 & $\mathrm{CuCN}$ & $\mathrm{Yb}(\mathrm{OTf})_{3}(1$ equiv. $)$ & 48 & N.R. \\
\hline 18 & $\mathrm{Cu}_{2} \mathrm{O}$ & $\mathrm{Ni}(\mathrm{OAc})_{2} \bullet 4 \mathrm{H}_{2} \mathrm{O}$ (1 equiv. $)$ & 65 & 16 \\
\hline 19 & $\mathrm{Cu}(\mathrm{OAc})_{2}$ & $\mathrm{Co}\left(\mathrm{NO}_{3}\right)_{2} \cdot 6 \mathrm{H}_{2} \mathrm{O}$ (1 equiv. $)$ & 65 & Trace \\
\hline 20 & $\mathrm{CuCl}_{2}$ & $\mathrm{Cu}(\mathrm{OAc})_{2} \bullet 4 \mathrm{H}_{2} \mathrm{O}$ ( 1 equiv. $)$ & 65 & N.R. \\
\hline 21 & $\mathrm{CuCl}_{2}$ & $\mathrm{LiCl}$ (1 equiv.) & 49 & N.R. \\
\hline 22 & $\mathrm{CuCl}_{2}$ & $\mathrm{CaCl}_{2}$ (1 equiv.) & 49 & 23 \\
\hline 23 & $\mathrm{CuCl}_{2}$ & $\begin{array}{l}\mathrm{CoCl}_{2}(1 \text { equiv. })+ \\
\mathrm{PivOH}(1 \text { equiv. })\end{array}$ & 58 & 47 \\
\hline 24 & $\mathrm{CuCl}_{2}$ & $\begin{array}{l}\mathrm{CoCl}_{2}(1 \text { equiv. })+ \\
\mathrm{PhCOOH}(1 \text { equiv. })\end{array}$ & 57 & 69 \\
\hline 25 & $\mathrm{CuCl}_{2}$ & $\begin{array}{l}\mathrm{CoCl}_{2}(20 \mathrm{~mol} \%)+ \\
\mathrm{PhCOOH}(20 \mathrm{~mol} \%)\end{array}$ & 84 & 78 \\
\hline 26 & None & $\begin{array}{l}\mathrm{CoCl}_{2}(1 \text { equiv. })+ \\
\mathrm{PhCOOH}(20 \mathrm{~mol} \%)\end{array}$ & 77 & N.R. \\
\hline
\end{tabular}

${ }^{a}$ Conditions: 1a (50 mg, $\left.0.28 \mathrm{mmol}\right), \mathrm{CuCl}_{2}(20 \mathrm{~mol} \%)$, additive (1 equiv.), THF (1.2 mL), $\mathrm{O}_{2}, 100{ }^{\circ} \mathrm{C} .{ }^{b}$ Isolated yield.

With the optimized reaction conditions in hand, the generality and limitations of this $\mathrm{Cu}$-catalyzed decarbonylation cyclization for forming isatins were explored (Table 2). A series of electron-donating and electron-withdrawing substitutents on the aromatic ring was examined. It was found that these substrates could be successfully converted to the corresponding isatins in moderate to good yields. Isatin derivatives $\mathbf{2 f}$ and $\mathbf{2 g}$ containing $\mathrm{Br}$ and $\mathrm{F}$ atoms were smoothly obtained in $71 \%$ and $56 \%$ yields, respectively, which had potential application for further functionalization into some bioactive compounds (Table 2). It is worth noting that electron-withdrawing $\mathrm{CF}_{3}$ and COOMe substitutents at the meta position on the aromatic rings were tolerated well to furnish the products $\mathbf{2 h}$ and $\mathbf{2 i}$ in moderate yields. To the best of our knowledge, the previous approach reported by Cheng et al. ${ }^{[7 \mathrm{~g}]}$ could not synthesize these isatins. Accordingly, a complementary access to isatin derivatives with electron-withdrawing groups was offered. In addition to examining the substituents on the aromatic ring, the substrates with different size chain on nitrogen atom were also investigated. As expected, substrates with $\mathrm{Et}, \mathrm{Bn}, n$-Bu on nitrogen efficiently underwent the $\mathrm{Cu}$-catalyzed annulation to give the corresponding isatins in $54 \% \sim 69 \%$ yields (Table 2). To our surprise, $\mathrm{N}$-(2-acetylphenyl)formamide without methyl group on the nitrogen also worked well under the standard conditions and provided the desired product $\mathbf{2 m}$ in $48 \%$ yield (Table 2).

Table 2 Scope of substrates for the synthesis of isatins ${ }^{a}$
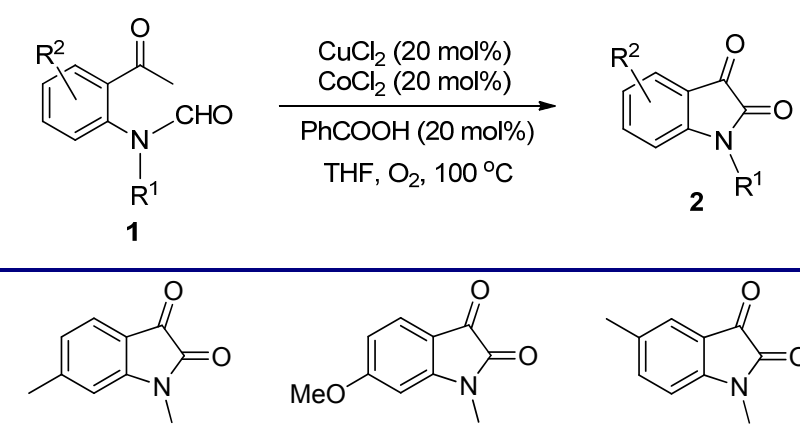<smiles>Cc1ccc2c(c1)C(=O)C(=O)N2C</smiles><smiles>COc1ccc2c(c1)C(=O)C(=O)N2C</smiles>
2c $44 \%$

2d $61 \%$<smiles>CN1C(=O)C(=O)c2cc(Br)ccc21</smiles><smiles>CN1C(=O)C(=O)c2cc(F)ccc21</smiles>

2g, $56 \%$<smiles>COC(=O)c1ccc2c(c1)N(C)C(=O)C2=O</smiles>

2h, $45 \%$

$2 \mathrm{i}, 50 \%$<smiles>CCN1C(=O)C(=O)c2ccccc21</smiles><smiles>CCCCN1C(=O)C(=O)c2ccccc21</smiles><smiles>O=C1C(=O)N(Cc2ccccc2)c2ccccc21</smiles>

2I, $62 \%$<smiles>O=C1Nc2ccccc2C1=O</smiles>

$2 \mathrm{~m}, 48 \%$ 2k, $54 \%$

${ }^{a}$ Conditions: 1 (0.3 mmol), $\mathrm{CuCl}_{2}(20 \mathrm{~mol} \%), \mathrm{CoCl}_{2}(20 \mathrm{~mol} \%), \mathrm{PhCOOH}(20$ $\mathrm{mol} \%)$, THF (1.2 mL), $\mathrm{O}_{2}, 100{ }^{\circ} \mathrm{C}$. Isolated yield.

Substrates 1p and 1q were also subjected to the standard conditions. Substrate 1p did not work. Substrate 1q could give the desired product $\mathbf{2 a}$ in $33 \%$ yield after $131 \mathrm{~h}$. These substrates did not show good reactivity (Scheme 2).

To gain insight into the reaction mechanism, some mechanistic studies were conducted. Compound ln bearing 


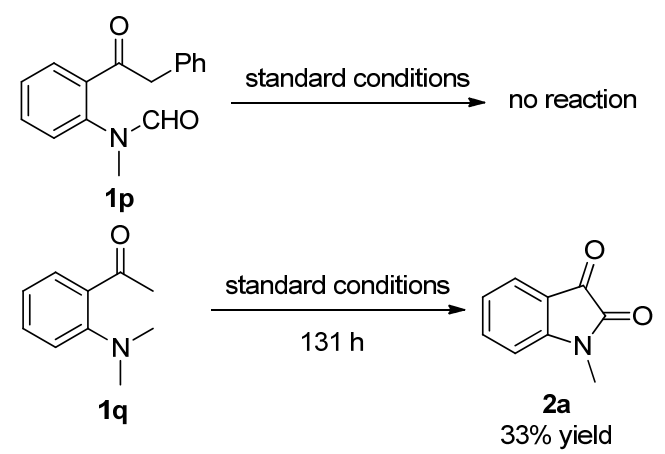

Scheme 2 Reaction of compounds 1p and 1q in standard conditions

${ }^{13} \mathrm{C}$ isotope on aldehyde group was prepared, and $\mathbf{1 n}$ was subjected to the standard conditions (Eq. 1). It was found that the carbon of amide in $\mathbf{2 a}$ came from methyl group, not aldehyde. In addition, it was found that the substrate 10 without aldehyde on nitrogen was performed sluggishly under $\mathrm{Cu}$ catalytic conditions to furnish the corresponding product 2 a with low $29 \%$ yield (Eq. 2). It was worth noting that an intermediate was discovered during the course of reaction, which was determined as a dichloronated compound 3a by the analysis of ${ }^{1} \mathrm{H} /{ }^{13} \mathrm{C}$ NMR and HRMS. To our surprise, compound 3a smoothly converted into the deserted product 2a under the standard conditions $(75 \%$ yield, Eq. 3), which revealed that compound 3a was a key intermediate in this reaction. Consequently, the reaction profile using 1a as the model substrate was obtained. It was found that the desired product $\mathbf{2 a}$ was produced with the formation of intermediate $\mathbf{3 a}$ (Figure 1). It further supported dichloronated compound $\mathbf{3 a}$ as a key intermediate in this reaction. When a radical trapping agent such as 2,2,6,6-tetramethyl-1-piperidinyloxy free radical (TEMPO) (20 $\mathrm{mol} \%$ ) was added into the reaction, no reaction occurred (Eq. 4). However, although the mechanism for the present catalytic reaction is not clear, a radical process for this reaction can be possible.
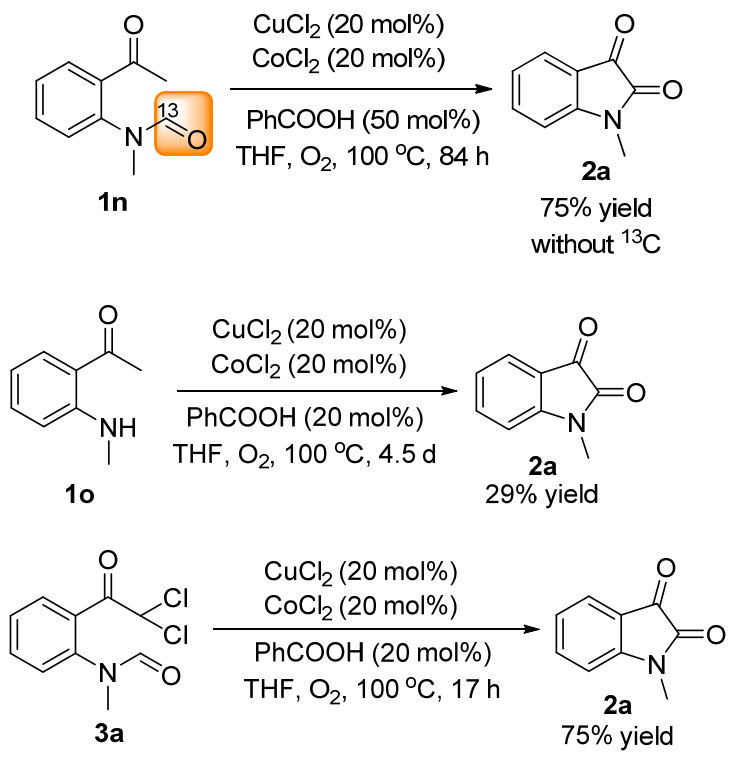

(1)<smiles>[R]Nc1ccc(C(C)C)cc1C(=O)C(Cl)(Cl)C(Cl)(Cl)Cl</smiles><smiles>[R]N1C(=O)C(=O)c2ccccc21</smiles>

Scheme 3 Postulated mechanism for the formation of isatins

\section{Conclusion}

In summary, a copper-catalyzed decarbonylation cyclization to form isatins using oxygen as a terminal oxidant was developed. This complementary way offers a new 
protocol for the synthesis of isatins through $\mathrm{C}\left(\mathrm{sp}^{3}\right)-\mathrm{H}$ bond functionalization in $\mathrm{Cu} / \mathrm{O}_{2} / \mathrm{Co}$ system. This system shows good reactivity and compatibility. Both electron-rich and electron-deficient functional groups can be tolerated. Further exploration of the generality and application of this approach is ongoing in our laboratory.

\section{Experimental section}

\subsection{General considerations}

All experiments were carried out under an oxygen atmosphere unless otherwise noted. Reactions were monitored using thin-layer chromatography (TLC). ${ }^{1} \mathrm{H}$ NMR and ${ }^{13} \mathrm{C}$ NMR spectra were obtained at 400 and $100 \mathrm{MHz}$, respectively on a Mercury Plus-400. NMR spectra were run in a solution of deuterated chloroform $\left(\mathrm{CDCl}_{3}\right)$. High-resolution mass spectra (HRMS) were recorded using electrospray ionization (ESI) with a Q-TOF MS Spectrometer. The substrates were prepared according to the reported literatures. ${ }^{[13,14]}$

\subsection{General procedure for Cu-catalyzed decar-} bonylation cyclization for the synthesis of isatins

An oven-dried Schlenk tube equipped with a magnetic stir bar was evacuated and backfilled with oxygen three times. Under oxygen, substrates $1(0.3 \mathrm{mmol}), \mathrm{CuCl}_{2}(20$ $\mathrm{mol} \%$, $0.06 \mathrm{mmol}, 8.1 \mathrm{mg}), \mathrm{CoCl}_{2}(20 \mathrm{~mol} \%$, $0.06 \mathrm{mmol}$, $7.8 \mathrm{mg}$ ), $\mathrm{PhCOOH}(20 \mathrm{~mol} \%, 0.06 \mathrm{mmol}, 7.3 \mathrm{mg}$ ) and tetrahydrofuran (THF) $(1.2 \mathrm{~mL})$ were added into the tube. Then the reaction was stirred at $100{ }^{\circ} \mathrm{C}$ for specific time. THF was removed under reduced pressure. Then, the mixture was extracted with EtOAc $(10 \mathrm{~mL} \times 3)$. The combined organic layers were dried over $\mathrm{Na}_{2} \mathrm{SO}_{4}$, filtered and concentrated in vacuo. The residue was purified by column chromatography to give the corresponding isatin products. All of products are known compounds. ${ }^{[14 \sim 18]}$

1-Methylindoline-2,3-dione (2a): ${ }^{[15]} \quad 78 \%$ yield. ${ }^{1} \mathrm{H}$ NMR (400 MHz, $\left.\mathrm{CDCl}_{3}\right) \delta: 3.26(\mathrm{~s}, 3 \mathrm{H}), 6.91(\mathrm{~d}, J=8.0$ $\mathrm{Hz}, 1 \mathrm{H}), 7.16(\mathrm{td}, J=1.2,7.6 \mathrm{~Hz}, 1 \mathrm{H}), 7.62 \sim 7.59(\mathrm{~m}$, $2 \mathrm{H}$ ); HRMS (ESI) calcd for $\mathrm{C}_{9} \mathrm{H}_{8} \mathrm{NO}_{2}$ 162.0555, found 162.0544 .

1,6-Dimethylindoline-2,3-dione $(\mathbf{2 b})::^{[15]} 62 \%$ yield. ${ }^{1} \mathrm{H}$ NMR (400 MHz, $\left.\mathrm{CDCl}_{3}\right) \delta: 2.45(\mathrm{~s}, 3 \mathrm{H}), 3.23(\mathrm{~s}, 3 \mathrm{H}), 6.69$ (s, 1H), 6.93 (d, $J=7.6 \mathrm{~Hz}, 1 \mathrm{H}), 7.50$ (d, $J=7.6 \mathrm{~Hz}, 1 \mathrm{H})$; HRMS (ESI) calcd for $\mathrm{C}_{10} \mathrm{H}_{10} \mathrm{NO}_{2}$ 176.0712, found 176.0704 .

6-Methoxy-1-methylindoline-2,3-dione (2c): ${ }^{[16]} \quad 44 \%$ yield. ${ }^{1} \mathrm{H}$ NMR (400 MHz, $\left.\mathrm{CDCl}_{3}\right) \delta: 3.22$ (s, 3H), 3.93 (s, $3 \mathrm{H}), 6.37(\mathrm{~d}, J=2.4 \mathrm{~Hz}, 1 \mathrm{H}), 6.57$ (dd, $J=8.4,2.0 \mathrm{~Hz}$, $1 \mathrm{H}), 7.59(\mathrm{~d}, J=8.4 \mathrm{~Hz}, 1 \mathrm{H})$; HRMS (ESI) calcd for $\mathrm{C}_{10} \mathrm{H}_{10} \mathrm{NO}_{3}$ 192.0661, found 192.0647.

1,5-Dimethylindoline-2,3-dione (2d): ${ }^{[15]} 61 \%$ yield; ${ }^{1} \mathrm{H}$ NMR (400 MHz, $\left.\mathrm{CDCl}_{3}\right) \delta: 2.33(\mathrm{~s}, 3 \mathrm{H}), 3.22(\mathrm{~s}, 3 \mathrm{H}), 6.80$ (d, $J=7.6 \mathrm{~Hz}, 1 \mathrm{H}), 7.41 \sim 7.38(\mathrm{~m}, 2 \mathrm{H})$; HRMS (ESI) calcd for $\mathrm{C}_{10} \mathrm{H}_{10} \mathrm{NO}_{2}$ 176.0712, found 176.0700.

5-Methoxy-1-methylindoline-2,3-dione (2e): ${ }^{[15]} \quad 65 \%$ yield; ${ }^{1} \mathrm{H}$ NMR $\left(400 \mathrm{MHz}, \mathrm{CDCl}_{3}\right) \delta: 3.24(\mathrm{~s}, 3 \mathrm{H}), 3.82(\mathrm{~s}$, $3 \mathrm{H}), 6.84(\mathrm{~d}, J=8.4 \mathrm{~Hz}, 1 \mathrm{H}), 7.19 \sim 7.15$ (m, 2H); HRMS
(ESI) calcd for $\mathrm{C}_{10} \mathrm{H}_{10} \mathrm{NO}_{3}$ 192.0661, found 192.0651 .

5-Bromo-1-methylindoline-2,3-dione (2f): $:^{[15]} 71 \%$ yield; ${ }^{1} \mathrm{H}$ NMR (400 MHz, $\left.\mathrm{CDCl}_{3}\right) \delta: 3.25(\mathrm{~s}, 3 \mathrm{H}), 6.82$ (dd, $J=$ $7.2,1.6 \mathrm{~Hz}, 1 \mathrm{H}), 7.71(\mathrm{~s}, 1 \mathrm{H}), 7.74(\mathrm{dd}, J=7.6,2.0 \mathrm{~Hz}$, $1 \mathrm{H}$ ); HRMS (ESI) calcd for $\mathrm{C}_{9} \mathrm{H}_{7} \mathrm{NO}_{3} \mathrm{Br} 239.9660$, found 239.9640 .

5-Fluoro-1-methylindoline-2,3-dione $\quad(\mathbf{2 g}):^{[15]}$ Orange solid, $56 \%$ yield. m.p. $150.4 \sim 151.3{ }^{\circ} \mathrm{C}$ (lit. $150.7 \sim$ $\left.152.5{ }^{\circ} \mathrm{C}\right) ;{ }^{1} \mathrm{H}$ NMR (400 MHz, $\left.\mathrm{CDCl}_{3}\right) \delta: 3.26(\mathrm{~s}, 3 \mathrm{H})$, $6.88 \sim 6.85(\mathrm{~m}, 1 \mathrm{H}), 7.36 \sim 7.31(\mathrm{~m}, 2 \mathrm{H}) ; \mathrm{HRMS}(\mathrm{ESI})$ calcd for $\mathrm{C}_{9} \mathrm{H}_{7} \mathrm{NO}_{3} \mathrm{~F} 180.0461$, found 180.0454 .

1-Methyl-6-(trifluoromethyl)indoline-2,3-dione (2h): $:^{[17]}$ $45 \%$ yield. ${ }^{1} \mathrm{H}$ NMR (400 $\left.\mathrm{MHz}, \mathrm{CDCl}_{3}\right) \delta: 3.32$ (s, 3H), $7.11(\mathrm{~s}, 1 \mathrm{H}), 7.43(\mathrm{~d}, J=8.0 \mathrm{~Hz}, 1 \mathrm{H}), 7.73(\mathrm{~d}, J=8.0 \mathrm{~Hz}$, $1 \mathrm{H}) ;{ }^{13} \mathrm{C} \mathrm{NMR}\left(100 \mathrm{MHz}, \mathrm{CDCl}_{3}\right) \delta: 26.4,106.9(\mathrm{~d}, J=$ $3.5 \mathrm{~Hz}, 1 \mathrm{C}), 119.4,120.8$ (d, $J=3.7 \mathrm{~Hz}, 1 \mathrm{C}), 124.1,125.5$, $139.2(\mathrm{~d}, J=33.3 \mathrm{~Hz}, 1 \mathrm{C}), 151.5,157.4,182.4$; HRMS (ESI) calcd for $\mathrm{C}_{10} \mathrm{H}_{7} \mathrm{NO}_{2} \mathrm{~F}_{3} 230.0429$, found 230.0425.

1-Methyl-2,3-dioxoindoline-6-carboxylate $(\mathbf{2 i}):{ }^{[18]} 50 \%$ yield. ${ }^{1} \mathrm{H}$ NMR $\left(400 \mathrm{MHz}, \mathrm{CDCl}_{3}\right) \delta: 3.32(\mathrm{~s}, 3 \mathrm{H}), 3.98(\mathrm{~s}$, $3 \mathrm{H}), 7.54(\mathrm{~d}, J=1.2 \mathrm{~Hz}, 1 \mathrm{H}), 7.68(\mathrm{dd}, J=8.0,0.8 \mathrm{~Hz}$, $1 \mathrm{H}), 7.84$ (dd, $J=7.6,1.2 \mathrm{~Hz}, 1 \mathrm{H}) ;{ }^{13} \mathrm{C}$ NMR $(100 \mathrm{MHz}$, $\left.\mathrm{CDCl}_{3}\right) \delta: 26.4,52.9,110.6,120.0,125.2,138.5,151.1$, 157.6, 165.2, 183.1; HRMS (ESI) calcd for $\mathrm{C}_{11} \mathrm{H}_{10} \mathrm{NO}_{4}$ 220.0610 , found 220.0604 .

1-Ethylindoline-2,3-dione (2j): $:{ }^{[19]} 69 \%$ yield. ${ }^{1} \mathrm{H}$ NMR $\left(400 \mathrm{MHz}, \mathrm{CDCl}_{3}\right) \delta: 1.34(\mathrm{t}, J=7.2 \mathrm{~Hz}, 1 \mathrm{H}), 3.82$ (q, $J=$ $7.2 \mathrm{~Hz}, 2 \mathrm{H}), 6.92$ (d, $J=7.6 \mathrm{~Hz}, 1 \mathrm{H}), 7.13(\mathrm{td}, J=0.8,7.2$ $\mathrm{Hz}, 1 \mathrm{H}), 7.62 \sim 7.57(\mathrm{~m}, 2 \mathrm{H})$; HRMS (ESI) calcd for $\mathrm{C}_{10} \mathrm{H}_{10} \mathrm{NO}_{2}$ 176.0712, found 176.0700.

1-Butylindoline-2,3-dione (2k): ${ }^{[20]} 54 \%$ yield. ${ }^{1} \mathrm{H}$ NMR $\left(400 \mathrm{MHz}, \mathrm{CDCl}_{3}\right) \delta: 0.99(\mathrm{t}, J=7.2 \mathrm{~Hz}, 3 \mathrm{H}), 1.46 \sim 1.37$ $(\mathrm{m}, 2 \mathrm{H}), 1.72 \sim 1.65(\mathrm{~m}, 2 \mathrm{H}), 3.74(\mathrm{t}, J=7.2 \mathrm{~Hz}, 2 \mathrm{H}), 6.91$ $(\mathrm{d}, J=8.0 \mathrm{~Hz}, 1 \mathrm{H}), 7.13(\mathrm{dt}, J=7.6,0.8 \mathrm{~Hz}, 1 \mathrm{H}), 7.61 \sim$ $7.56(\mathrm{~m}, 2 \mathrm{H})$; HRMS (ESI) calcd for $\mathrm{C}_{12} \mathrm{H}_{14} \mathrm{NO}_{2} 204.1025$, found 204.1011 .

1-Benzylindoline-2,3-dione (2l): ${ }^{[15]} 62 \%$ yield. ${ }^{1} \mathrm{H}$ NMR $\left(400 \mathrm{MHz}, \mathrm{CDCl}_{3}\right) \delta: 4.93(\mathrm{~s}, 2 \mathrm{H}), 6.78(\mathrm{~d}, J=8.0 \mathrm{~Hz}$, $1 \mathrm{H}), 7.11(\mathrm{t}, J=7.6 \mathrm{~Hz}, 1 \mathrm{H}), 7.37 \sim 7.30(\mathrm{~m}, 5 \mathrm{H}), 7.50(\mathrm{dt}$, $J=7.6,0.8 \mathrm{~Hz}, 1 \mathrm{H}), 7.62(\mathrm{~d}, J=7.6 \mathrm{~Hz}, 1 \mathrm{H})$; HRMS (ESI) calcd for $\mathrm{C}_{15} \mathrm{H}_{12} \mathrm{NO}_{2} 238.0868$, found 238.0858.

Indoline-2,3-dione (2m): ${ }^{[15]} 48 \%$ yield; ${ }^{1} \mathrm{H}$ NMR (400 $\left.\mathrm{MHz}, \mathrm{CDCl}_{3}\right) \delta: 6.93(\mathrm{~d}, J=8.0 \mathrm{~Hz}, 1 \mathrm{H}), 7.15(\mathrm{td}, J=0.8$, $7.6 \mathrm{~Hz}, 1 \mathrm{H}), 7.59(\mathrm{td}, J=1.2,7.6 \mathrm{~Hz}, 1 \mathrm{H}), 7.63(\mathrm{~d}, J=7.6$ $\mathrm{Hz}, 1 \mathrm{H}$ ), 8.09 (br s, 1H); HRMS (ESI) calcd for $\mathrm{C}_{8} \mathrm{H}_{6} \mathrm{NO}_{2}$ 148.0399, found 148.0386.

$N$-(2-Acetylphenyl)- $N$-methylformamide- ${ }^{13} \mathrm{C}$ (1n): Major : Minor $=3: 1 ;{ }^{1} \mathrm{H} \mathrm{NMR}\left(400 \mathrm{MHz}, \mathrm{CDCl}_{3}\right) \delta$ : Major: $2.51(\mathrm{~s}, 3 \mathrm{H}), 3.26(\mathrm{~s}, 3 \mathrm{H}), 7.25(\mathrm{dd}, J=1.2,7.2 \mathrm{~Hz}, 1 \mathrm{H})$, $7.43(\mathrm{td}, J=1.2,7.6 \mathrm{~Hz}, 1 \mathrm{H}), 7.57(\mathrm{td}, J=1.6,7.6 \mathrm{~Hz}, 1 \mathrm{H})$, 7.70 (dd, $J=7.6,1.6 \mathrm{~Hz}, 1 \mathrm{H}), 8.38(\mathrm{~s}, 1 \mathrm{H})$; Minor: $2.56(\mathrm{~s}$, $3 \mathrm{H}), 3.37$ (s, 3H), $7.24(\mathrm{~d}, J=7.2 \mathrm{~Hz}, 1 \mathrm{H}), 7.38$ (td, $J=$ $1.2,7.6 \mathrm{~Hz}, 1 \mathrm{H}) 7.53(\mathrm{td}, J=1.6,7.6 \mathrm{~Hz}, 1 \mathrm{H}), 7.65$ (dd, $J=1.2,7.6 \mathrm{~Hz}, 1 \mathrm{H}), 8.40(\mathrm{~s}, 1 \mathrm{H}) ;{ }^{13} \mathrm{C} \mathrm{NMR}(100 \mathrm{MHz}$, $\left.\mathrm{CDCl}_{3}\right) \delta: 29.5,33.8,128.0,129.8,132.7,136.4,162.6$, 199.6; HRMS (ESI) calcd for $\mathrm{C}_{10} \mathrm{H}_{12} \mathrm{NO}_{2}$ 178.0902, found 178.0891 . 
$N$-(2-(2,2-Dichloroacetyl)phenyl)- $N$-methylformamide (3a): Major : Minor $=3: 1 ;{ }^{1} \mathrm{H}$ NMR $\left(400 \mathrm{MHz}, \mathrm{CDCl}_{3}\right)$ $\delta$ : Major: 3.25 (s, 3H), $6.48(\mathrm{~s}, 1 \mathrm{H}), 7.33(\mathrm{~d}, J=7.6 \mathrm{~Hz}$, $1 \mathrm{H}), 7.52$ (t, $J=7.6 \mathrm{~Hz}, 1 \mathrm{H}), 7.67$ (t, $J=7.6 \mathrm{~Hz}, 1 \mathrm{H}), 7.84$ $(\mathrm{d}, J=7.6 \mathrm{~Hz}, 1 \mathrm{H}), 8.19$ (s, 1H); Minor: 3.39 (s, 3H), 6.51 (s, 1H), 7.30 (d, $J=8.0 \mathrm{~Hz}, 1 \mathrm{H}), 7.44$ (t, $J=8.0 \mathrm{~Hz}, 1 \mathrm{H})$, $7.64(\mathrm{t}, J=8.0 \mathrm{~Hz}, 1 \mathrm{H}), 7.91(\mathrm{~d}, J=8.0 \mathrm{~Hz}, 1 \mathrm{H}), 8.18$ (s, $1 \mathrm{H}) ;{ }^{13} \mathrm{C}$ NMR $\left(100 \mathrm{MHz}, \mathrm{CDCl}_{3}\right) \delta$ : Major: 34.6, 69.3, $128.7,129.5,130.4,132.1,134.5,141.8,162.8,188.1$; Minor: 38.0, 69.0, 127.9, 128.1, 129.3, 132.7, 134.0, 139.1, 163.1, 188.3; HRMS (ESI) calcd for $\mathrm{C}_{10} \mathrm{H}_{9} \mathrm{Cl}_{2} \mathrm{NO}_{2} \mathrm{Na}$ $\left(\mathrm{M}+\mathrm{Na}^{+}\right)$267.9908, found 267.9903 .

Supporting Information ${ }^{1} \mathrm{H}$ NMR spectra of all products and data of intermediate 3a. The Supporting Information is available free of charge via the Internet at http://siocjournal.cn.

\section{References}

[1] (a) Sumpter, W. C. Chem. Rev. 1944, 34, 393.

(b) da Silva, J. F. M.; Garden, S. J.; Pinto, A. C. J. Braz. Chem. Soc. 2001, 12, 273.

[2] (a) Ratan, B. T.; Anand, B.; Yogeeswari, P.; Sriram, D. Bioorg. Med. Chem. Lett. 2005, 15, 4451.

(b) Raj, A.; Raghunathan, R.; Sridevikumaria, M. R.; Raman, N. Bioorg. Med. Chem. 2003, 11, 407.

(c) Verma, M.; Pandeya, S. N.; Singh, K. N.; Stables, J. P. Acta Pharm. 2004, 54, 49.

(d) Jiang, T.; Kuhen, K. L.; Wolff, K.; Yin, H.; Bieza, K.; Caldwell, J.; Bursulaya, B.; Tuntlad, T.; Zhang, K.; Karanewsky, D.; He, Y. Bioorg. Med. Chem. Lett. 2006, 16, 2109.

(e) Aboul-Fadl, T.; Bin-Jubair, F. A. S. Int. J. Res. Pharm. Sci. 2010, 1,113 .

(f) Sharma, S.; Gupta, M. K.; Saxena, A. K.; Bedi, P. M. S. Bioorg. Med. Chem. 2015, 23, 7165 .

(g) Harbinder, S.; Jatinder, V. S.; Gupta, M. K.; Sharma, S.; Nepali, K.; Bedi, P. M. S. Bioorg. Med. Chem. Lett. 2017, 27, 3974.

[3] Sandmeyer, T. Helv. Chim. Acta 1919, 2, 234.

[4] (a) Stollé, R. Ber. Dtsch. Chem. Ges. 1913, 46, 3915.

(b) Stollé, R. J. Prakt. Chem. 1922, 106, 137.

[5] (a) Martinet, J. Compt. Rend. 1918, 166, 85.

(b) Bonnefoy, J.; Martinet, J. Compt. Rend. 1921, 172, 220.

[6] Xie, Y. Chem. Commun. 2016, 52, 12372.

[7] (a) Sun, J.; Liu, B.; Xu, B. RSC Adv. 2013, 3, 5824.

(b) Liu, T.; Yang, H.; Jiang, Y.; Fu, H. Adv. Synth. Catal. 2013, 355, 1169 . (c) Tang, B.-X.; Song, R.-J.; Wu, C.-Y.; Liu, Y.; Zhou, M.-B.; Wei, W.-T.; Deng, G.-B.; Yin, D.-L.; Li, J.-H. J. Am. Chem. Soc. 2010, 132,8900

(d) Liu, T.; Yang, H.; Jiang, Y.; Fu, H. Adv. Synth. Catal. 2013, 355, 1169.

(e) Sun, J.; Liu, B.-X.; Xu, B. RSC Adv. 2013, 3, 5824.

(f) Ilangovan, A.; Satish, G. Org. Lett. 2013, 15, 5726.

(g) Huang, P. C.; Gandeepan, P.; Cheng, C. H. Chem. Commun. 2013, 49, 8540.

(h) Li, J.; Zheng, Y.; Yu, X. L.; Lv, S. Y.; Wang, Q. T.; Hai, L.; Wu Y. RSC Adv. 2015, 5, 103280.

(i) Liu, Z.; Zhang, J.; Chen, S.; Shi, E.; Xu, Y.; Wan, X. Angew. Chem., Int. Ed. 2012, 51, 3231.

(j) Meng, Q.; Wang, F.; Li, M. J. Mol. Model. 2013, 19, 2225.

[8] Liao, Y.-Y.; Gao, Y.-C.; Zheng, W.; Tang, R.-Y. Adv. Synth. Catal. 2018, 360, 3391.

[9] Lollar, C. T.; Krenek, K. M.; Bruemmer, K. J.; Lippert, A. R. Org. Biomol. Chem. 2014, 12, 406.

[10] (a) Satish, G.; Polu, A.; Ramar, T.; Ilangovan, A. J. Org. Chem. 2015, 80, 5167.

(b) Reddy, M. R.; Rao, N. N.; Ramakrishna, K.; Meshram, H. M. Tetrahedron Lett. 2014, 55, 4758

(c) Ilangovan, A.; Satish, G. J. Org. Chem. 2014, 79, 4984.

(d) Gao, F. F.; Xue, W. J.; Wang, J. G.; Wu, A. X. Tetrahedron 2014 $70,4331$.

[11] (a) Huang, P. C.; Gandeepan, P.; Cheng, C. H. Chem. Commun. 2013, 49,8540 .

(b) Wu, H.; Zhang, Z. G.; Liu, Q. F.; Liu, T. X.; Ma, N. N.; Zhang, G. S. Org. Lett. 2018, 20, 2897.

(c) Salvanna, N.; Reddy, L. M.; Kumar, R. A.; Das, B. ChemistrySelect 2018, 3, 8019.

[12] Nobrega, J. A.; Goncalves, S. M. C.; Peppe, C. Synth. Commun. 2002, 32, 3711.

[13] Shekhar, A. C.; Kumar, A. R.; Sathaiah, G.; Paul, V. L.; Sridhar, M.; Rao, P. S. Tetrahedron Lett. 2009, 50, 7099.

[14] Kirincich, S. J.; Xiang, J.; Green, N.; Tam, S.; Yang, H. Y.; Shim, J.; Clark, J. D.; McKew, J. C. Bioorg. Med. Chem. 2009, 17, 4383.

[15] Luo, J. F.; Gao, S. S.; Ma, Y. R.; Ge, G. P. Synlett 2018, 29, 969

[16] Ji, H. H.; Zhu, Y. Z.; Shao, Y.; Liu, J.; Yuan, Y.; Jia, X. D. J. Org. Chem. 2017, 82, 9859.

[17] Wang, H. Y.; Wang, K. Y.; Man, Y. Q.; Gao, X. N.; Yang, L. M.; Ren, Y. F.; Li, N.; Tang, B.; Zhao, G. Adv. Synth. Catal. 2017, 359, 3934.

[18] Bredenkampa, A.; Mohrb, F.; Kirsch, S. F. Synthesis 2015, 47, 1937.

[19] Zhang, C.; Li, S.; Filip, B.; Richmond, L.; Ye, X.; Jiang, Z. ACS Catal. 2016, 6, 10, 6853.

[20] Salvanna, N.; Ramesh, P.; Kumarc, K. S.; Das, B. New J. Chem. 2017, 41, 13754. 